\title{
TMD Fragmentation Function at NNLO
}

\author{
Alexey A.Vladimirov ${ }^{* \dagger}$ \\ Institut für Theoretische Physik, Universität Regensburg, D-93040 Regensburg, Germany \\ E-mail: vladimirov.aleksev@gmail.com
}

\begin{abstract}
The calculation of the unpolarized non-singlet transverse momentum dependent fragmentation function (TMDFF) at next-to-next-to-leading order (NNLO) is presented. The calculation is done within the framework of the TMD factorization theorem by separate evaluation of the soft factor and TMD collinear correlator. For the fist time the TMD factorization theorem is confirmed at NNLO, which is a very strong check of the whole formalism. Using the result of calculation we extract the matching coefficient onto integrated fragmentation function. Also we extract NNLO anomalous dimensions, necessary to perform the transverse momentum resummation at nextto-next-to-next-to-leading-logarithmic accuracy, that is the first time direct evaluation of these anomalous dimension.
\end{abstract}

QCD Evolution 2015 -QCDEV2015-

26-30 may 2015

Jefferson Lab (JLAB), Newport News Virginia, USA

\footnotetext{
* Speaker.

${ }^{\dagger}$ In collaboration with M.G. Echevarria and I.Scimemi
} 


\section{Introduction}

Within recent years the interest to the transverse momentum dependent (TMD) distributions, first introduced in [U, ㅁ], constantly increases. The TMD distributions are the important objects for description of double-inclusive processes, such as Drell-Yan, Vector Boson/Higgs Production, Semi-Inclusive Deep Inelastic Scattering (SIDIS) and $e^{+} e^{-} \rightarrow 2$ hadrons, at moderate transverse momentum. All these processes are fundamental for current and future planned high energy colliders. For the review of recent status see [B]].

The formulation and proof of the TMD factorization theorem is complected. The typical expression for the hadronic tensor takes the form (here for SIDIS)

$$
W^{\mu v}\left(Q, q_{T}\right)=H^{\mu v}(Q) \int \frac{d^{2} b_{T}}{(2 \pi)^{2}} e^{-i q_{T} b_{T}} \Delta_{f}\left(x_{1}, b_{T}\right) \Delta_{d}\left(x_{2}, b_{T}\right) S\left(b_{T}\right)+Y,
$$

where $H$ is a hard coefficient function, $\Delta_{f}$ is the naive TMD parton distribution function (TMD$\mathrm{PDF}), \Delta_{d}$ is the naive TMD fragmentation function (TMDFF) and $S$ is the soft factor. The structure of TMD factorization theorem is schematically shown in fig】(left). For the following discussion it is important to stress that individually the functions $\Delta$ and $S$ are undefined, although the operator expressions for these objects are known. That happens due to the presence of rapidity divergences in every term of $(\mathbb{L})$. In the product of of components $(\mathbb{L})$ rapidity divergences cancel, that is

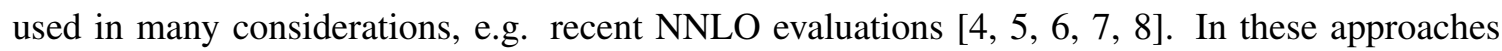
one evaluates the TMD cross-section directly on the integrated parton densities, see fig.W(right). In contrast, we consider the rapidity-divergences-free definition of individual TMD distributions.

The individual TMD distributions can be define with the help of non-Abelian exponentiation theorem applied to the soft factor. It allows effectively split the soft factor onto a product of two functions. The latters in the combination with TMD distributions $\Delta$ form a well-defined TMD distribution (see detailed description in [Q, 四, 四]). Due to the universality of soft factor for different processes, such an approach allows one to describe all TMD processes by universal TMDPDFs and TMDFFs. The discussed approach has been checked at one-loop for TMDs with various quantum

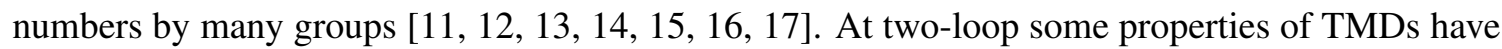

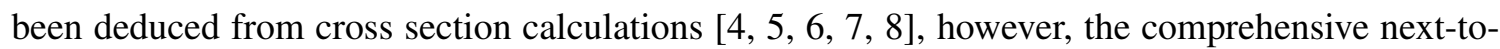
next-to-leading-order (NNLO) analysis has not been done so far. In this proceedings we discuss the details of the recent NNLO evaluation of the unpolarized TMDFF made by our group [20].

The evaluation of individual TMDs at higher orders is an utterly non-trivial check for their definition, since starting from the two-loop order, the singularities of various types mix up. Due to this fact, the choice of the regularization procedure is very important. The regularization scheme should be implemented in such a way that it takes care about ultraviolet (UV), mass, collinear and rapidity divergences in the distinguishable manner. Each type of the divergences is to be cancel by appropriate procedure: renormalization procedure takes care about UV divergences, mass divergences cancel in the sum of virtual and real graphs, rapidity divergences cancel in the product of TMD definition components. The rest collinear divergences are the part of low-energy hadron structure, and can be absorbed into the non-perturbative parton density by matching procedure. Within the discussed calculation [20] the convenient regularization scheme was elaborated and the cancelation of all divergences in the appropriate sectors has been checked. 

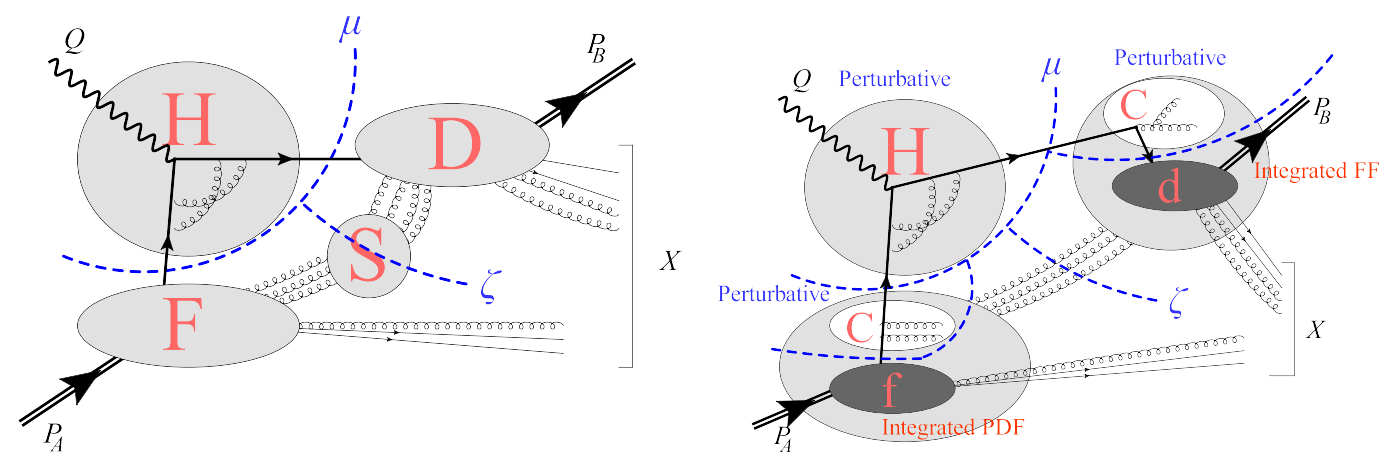

Figure 1: Illustration of TMD factorization theorems for SIDIS is presented. The gray blobs denote the factorized parts of perturbative series, hard coefficient function $(\mathrm{H})$, TMD distributions (F and D) and soft factor $(\mathrm{S})$. The dashed lines depict the factorization procedure and and adjusted by scales of corresponding factorization. The right frame shows the general structure of factorization theorem (1.1). The left frame shows the TMD factorization theorem with additional matching of TMD distributions on the integrated parton densities.

The main practical outcome of the NNLO calculation is the matching coefficient of TMDFF onto the integrated fragmentation function (FF) at NNLO. That allows to perform the NNLO resummations for inclusive processes and, thus, fills the gap within TMD phenomenology.

\section{Definitions}

The soft factor (SF) is a spin and process independent vacuum expectation value of Wilson lines [Q, 四], and it is defined as

$$
S\left(\mathbf{k}_{s \perp}\right)=\int \frac{d^{2} \mathbf{b}_{\perp}}{(2 \pi)^{2}} e^{i \mathbf{b}_{\perp} \cdot \mathbf{k}_{s \perp}} \frac{1}{N_{c}}\left\langle 0\left|\operatorname{Tr}\left[W_{n}^{T \dagger} \tilde{W}_{\bar{n}}^{T}\right]\left(0^{+}, 0^{-}, \mathbf{b}_{\perp}\right)\left[\tilde{W}_{\bar{n}}^{T^{\dagger}} W_{n}^{T}\right](0)\right| 0\right\rangle,
$$

where $W_{n}$ and $\tilde{W}_{\bar{n}}$ stand for Wilson lines along light-cone directions, and $n$ and $\bar{n}$ are light-cone vectors $\left(n^{2}=\bar{n}^{2}=0, n \cdot \bar{n}=2\right)$. The superscript $T$ on Wilson lines in (…) implies subsidiary transverse links from the light-cone infinities to the transverse infinity. These links guaranty gauge invariance and are necessary for calculations in singular gauges. Within the Feynman gauge, which is the gauge used for discussed calculation, the contribution of the transverse links vanish.

The unpolarized collinear matrix element in the position space defined as

$$
\begin{aligned}
& \Delta_{i \rightarrow h}^{(0)}\left(z, \mathbf{b}_{T}\right)= \\
& \frac{1}{2 z} \int \frac{d \xi^{-}}{2 \pi} e^{-i \xi^{+} P^{-}} \operatorname{Tr} \sum_{X} \int\left\langle 0\left|\left[\tilde{W}_{\bar{n}}^{T^{\dagger}} \psi\right]\left(\xi^{-}, 0^{-}, \mathbf{b}_{\perp}\right)\right| X ; P_{h}\right\rangle \not h /\left.\left\langle X ; P_{h}\left|\left[\bar{\psi}_{W_{\bar{b}}^{T}}^{T}\right](0)\right| 0\right\rangle\right|_{\text {s.s. }}
\end{aligned}
$$

where index $i$ refers to the parton flavor, $P$ is the hadron momentum, $z$ is the Bjorken variable. The subscript "s.s." stands for soft subtraction of matrix element. With in the Soft Collinear Effective Theory (SCET) literature it is known as "zero-bin" subtraction. These terms are equivalent in the present context. For the used regularization, soft subtraction is equivalent to division of the naively 
calculated colliner matrix element ([2.2) by the soft function in Eq. (ㅁ. $($ ). That forms the so-called "pure collinear" matrix element: $\Delta_{\text {pure }} \sim \Delta_{\text {naive }} S^{-1}$.

Individually both matrix elements ([2. $(1)$ and (2.2) have rapidity divergences at every order in the perturbative expansion. The essential property of the soft factor follows from the non-Abelian exponentiation of Wilson lines (see modern derivation in [[1]]). It is stated that the logarithm of the SF is maximally linear in the logarithmical rapidity divergences. Therefore, soft factor can be split into two pieces [प]],

$$
\tilde{S}\left(\mathbf{L}_{\mu}, \mathbf{L}_{\sqrt{\delta^{+} \delta^{-}}}\right)=\tilde{S}^{\frac{1}{2}}\left(\mathbf{L}_{\mu}, \mathbf{L}_{\delta^{+}}\right) \tilde{S}^{\frac{1}{2}}\left(\mathbf{L}_{\mu}, \mathbf{L}_{\delta^{-}}\right),
$$

where we introduce the convenient notation

$$
\mathbf{L}_{X}=\ln \left(X^{2} \mathbf{b}^{2} e^{2 \gamma_{E}} / 4\right)
$$

Variables $\delta^{ \pm}$are rapidity regulators that one uses in the $n$ - and $\bar{n}$-collinear sectors (our implementation of it is specified later in Eq. (B.D)). The result of the combination of one piece of the SF and the collinear correlator $(\Delta)$ is free from rapidity divergences and hence can be considered as a valid hadronic quantity. For the unpolarized TMDFF we have

$$
\tilde{D}_{i \rightarrow h}\left(z, \mathbf{L}_{\mu}, \mathbf{l}_{\zeta_{D}}\right)=Z_{D}\left(\mathbf{l}_{\zeta_{D}}\right) Z_{3}^{-1} \tilde{\Delta}_{i \rightarrow h}^{(0)}\left(z, \mathbf{L}_{\mu}, \mathbf{l}_{\zeta_{D}}, \mathbf{L}_{\delta^{+}}\right) \tilde{S}^{\frac{1}{2}}\left(\mathbf{L}_{\mu}, \mathbf{L}_{\delta^{+}}\right),
$$

where we have introduced the notation $\mathbf{l}_{\zeta}=\ln \left(\mu^{2} / \zeta\right)$. The factors $Z_{D}$ and $Z_{3}$ are the UV renormalization multipliers of the TMD operator and quark field respectively. The $\zeta_{F}$ and $\zeta_{D}$ are fractions of $Q^{2}$, satisfying $\zeta_{F} \zeta_{D}=Q^{4}$, where $\zeta_{F}=Q^{2} / \alpha$ and $\zeta_{D}=\alpha Q^{2}$ with $\alpha$ being arbitrary boost-invariant real number (in the following we omit the subscripts $F, D$ where unnecessary).

At small values of the impact parameter $\mathbf{b}$ the renormalized TMDFF can be factorized again in

$$
\tilde{D}_{i \rightarrow h}\left(z, \mathbf{L}_{\mu}, \mathbf{l}_{\zeta}\right)=\int_{z}^{1} \frac{d \tau}{\tau^{3-2 \varepsilon}} C_{i \rightarrow j}\left(\frac{z}{\tau}, \mathbf{L}_{\mu}, \mathbf{l}_{\zeta}\right) d_{j \rightarrow h}(\tau, \mu),
$$

where $d_{i \rightarrow h}(\xi, \mu)$ is the renormalized integrated FF. In Eq. (ㅁ.5) and in the rest of this letter. In this case the TMD factorization is illustrated in fig.W(right).

\section{Regularization}

The regularization scheme used for the calculation should satisfy several important demands, such as: it should respect the exponentiation property of Wilson lines; it should match the singularities of the naively calculated collinear matrix element in the soft limit with the ones of the SF. Additionally, the chosen regularization scheme should be convenient for multi-loop integral computations. The investigation of the popular schemes shows that they are inappropriate for NNLO evaluation, either violate some of demands, ether result to the overcomplecated two-loop integrals. Finally, our regularization scheme is build as following: We used the modified version the $\delta$-regularization, that has been derived from the $\delta$-regularization used by many authors, e.g. Refs. [], [3], [4, [प] ], to regularize the rapidity divergences. To regularize the rest of UV and infrared divergences we use standard dimensional regularization, while the incoming/outgoing partons are on-shell and massless. 
In order to match the required demands at multi-loop level we perform the following modifications of the $\delta$-regularization. First, in order to supply the non-abelian exponentiation, and hence the relation in $([2.3)$, the $\delta$-regulator should be implemented at the operator level, see e.g. the discussion in Ref. [18]]. With this purpose we modify the definition of Wilson line as

$$
\tilde{W}_{\bar{n}(n)}(0)=P \exp \left[-i g \int_{0}^{\infty} d \sigma A_{ \pm}(\sigma n)\right] \rightarrow P \exp \left[-i g \int_{0}^{\infty} d \sigma A_{ \pm}(\sigma n) e^{\delta^{ \pm}|\sigma|}\right],
$$

where $\delta^{ \pm} \rightarrow 0^{+}$. Second, in order to match the soft singularities of the naively calculated collinear matrix element and the soft factor, the $\delta^{ \pm}$in $\Delta_{i \rightarrow h}$ should be rescaled by factor $z$, i.e. $\delta \rightarrow \delta / z$. This rescaling is necessary for TMDFF, but not for TMDPDF. Besides the mathematical demands it can be explained by kinematical arguments. Therefore, in the modified $\delta$-regularization the Feynman rules for interaction with Wilson line became

$$
\frac{1}{\left(k_{1}^{+}-i 0\right)\left(k_{2}^{+}-i 0\right) \ldots\left(k_{n}^{+}-i 0\right)} \rightarrow \frac{1}{\left(k_{1}^{+}-i \delta^{+}\right)\left(k_{2}^{+}-2 i \delta^{+}\right) \ldots\left(k_{n}^{+}-n i \delta^{+}\right)},
$$

for soft factor and TMD PDF collinear matrix element, while for TMD FF collinear matrix element

$$
\frac{1}{\left(k_{1}^{+}-i 0\right)\left(k_{2}^{+}-i 0\right) \ldots\left(k_{n}^{+}-i 0\right)} \rightarrow \frac{1}{\left(k_{1}^{+}-i \delta^{+} / z\right)\left(k_{2}^{+}-2 i \delta^{+} / z\right) \ldots\left(k_{n}^{+}-n i \delta^{+} / z\right)} .
$$

Such modified regularization is appropriate for being used in multi-loop calculations and for the evaluation of the relevant matrix elements separately.

\section{Extraction of the matching coefficient}

The evaluation of TMD distribution consists in the evaluation of soft factor, the collinear matrix element and renormalization factors with subsequent recombination into equation (․2.2). At NLO the perturbative expression for TMDFF is

$$
\tilde{D}_{i \rightarrow j}^{[1]}=\tilde{\Delta}_{i \rightarrow j}^{[1]}-\frac{\tilde{S}^{[1]} \delta_{i j}}{2}+\left(Z_{D}^{[1]}-Z_{2}^{[1]}\right) \delta_{i j}
$$

where we use superscripts in square brackets to denote the order of the perturbative expansion, e.g. $S=\sum_{n} a_{s}^{n} S^{[n]}$, with $a_{s}=\frac{g^{2}}{(4 \pi)^{2}}$. The rapidity divergences arise in the first and the second terms, and cancel between them. The last two factors are pure ultraviolet poles. The obtained expression is singular at $\varepsilon \rightarrow 0$. These are standard collinear divergences, that are part of parton distribution. Comparing the perturbative expansion of the left- and right-hand-sides of equation ([2.5]), we obtain the following expression for the matching coefficient $C$ at NLO

$$
\tilde{C}_{i \rightarrow j}^{[1]}=\tilde{D}_{i \rightarrow j}^{[1]}-\frac{d_{i \rightarrow j}^{[1]}}{z^{2-2 \varepsilon}} .
$$

Note, that in our set of regularization all diagrams contributing to the integrated parton density are zero. Therefore, the function $d(z)$ consists of UV renormalization constant only. Thus, the UV singularity of $d(z)$ cancels the remain collinear singularity of (4.C), and we obtain the well known result [Q, 四]

$$
\tilde{C}_{q \rightarrow q}^{[1]}=\frac{C_{F} a_{s}}{z^{2}}\left[-2 \mathbf{L}_{\mu / z} \mathscr{P}_{q \rightarrow q}(z)+2 \bar{z}+\delta(\bar{z})\left(-\mathbf{L}_{\mu}^{2}+2 \mathbf{L}_{\mu} \mathbf{l}_{\zeta}+3 \mathbf{L}_{\mu}-\frac{\pi^{2}}{6}\right)\right],
$$


where $\mathscr{P}_{q \rightarrow q}(z)=\left(\left(1+z^{2}\right) /(1-z)\right)_{+}$is the quark splitting function. The plus-distribution is defined as $(f(z))_{+}=f(z)-\delta(\bar{z}) \int_{0}^{1} d y f(y)$.

The NNLO TMD reads

$$
\begin{aligned}
\tilde{D}_{i \rightarrow j}^{[2]}= & \tilde{\Delta}_{i \rightarrow j}^{[2]}-\frac{\tilde{S}^{[1]} \tilde{\Delta}_{i \rightarrow j}^{[1]}}{2}-\frac{\tilde{S}^{[2]} \delta_{i j}}{2}+\frac{3 \tilde{S}^{[1]} \tilde{S}^{[1]}}{8} \delta_{i j} \\
& +\left(Z_{D}^{[1]}-Z_{2}^{[1]}\right)\left(\tilde{\Delta}_{i \rightarrow j}^{[1]}-\frac{\tilde{S}_{+}^{[1]}}{2} \delta_{i j}\right)+\left(Z_{D}^{[2]}-Z_{2}^{[2]}-Z_{2}^{[1]} Z_{D}^{[1]}+Z_{2}^{[1]} Z_{2}^{[1]}\right) \delta_{i j}
\end{aligned}
$$

The first and the second lines are individually free of rapidity divergences. One can see that at two loops the structure of rapidity divergences cancelation is cumbersome. Explicit evaluation of all ingredients at NNLO confirm the cancelation of rapidity divergences and hence the correctness of TMD definition and NNLO.

The matching coefficient at two-loop level is given by the combination

$$
\tilde{C}_{i \rightarrow f}^{[2]}=\tilde{D}_{i \rightarrow f}^{[2]}-\tilde{C}_{i \rightarrow k}^{[1]} \otimes \frac{d_{k \rightarrow f}^{[1]}}{z^{2-2 \varepsilon}}-\frac{d_{i \rightarrow f}^{[2]}}{z^{2-2 \varepsilon}},
$$

where the symbol $\otimes$ denotes Mellin convolution in the Bjorken variable $z$, while $k$ is a flavor index.

\section{Renormalization group}

The renormalization group equations of the TMDFF and the integrated FF provide also important checks for our calculation. We have that

$$
\begin{aligned}
\mu^{2} \frac{d}{d \mu^{2}} \tilde{D}_{i \rightarrow h} & =\frac{1}{2} \gamma_{D}^{i} \tilde{D}_{i \rightarrow h} \\
\gamma_{D} & =\Gamma_{\text {cusp }}^{i} \mathbf{l}_{\zeta}-\gamma_{V}^{i},
\end{aligned}
$$

where $\Gamma_{\text {cusp }}$ is the cusp anomalous dimension. We also have an evolution in the rapidity parameter

$$
\begin{aligned}
\zeta \frac{d}{d \zeta} \tilde{D}_{i \rightarrow h} & =-\mathscr{D}^{i} \tilde{D}_{i \rightarrow h} \\
-2 \mu^{2} \frac{d}{d \mu^{2}} \mathscr{D}^{i} & =\zeta \frac{d}{d \zeta} \mathscr{D}^{i}=-\Gamma_{\text {cusp }}^{i} .
\end{aligned}
$$

Applying these equations on the definition of matching procedure (2.5) and using the DGLAP evolution for the integrated FF, the logarithmical part of matching coefficient can be predicted. Note, that at NLO no extra information is needed, while at NNLO one need the finite part of NLO calculation. Within our calculation we perform evaluation of all terms in the matching coefficient and we found complete agreement with the renormalization group predictions. That is a good check of the final result.

The anomalous dimension $\mathscr{D}$ is known up to two-loop order, and originally was extracted from the analysis of Drell-Yan processes. In fact our consideration is of more generic form, and allows to extract the function $\mathscr{D}$ for all TMD processes from the explicit expression for the soft factor. In this way we confirm that anomalous dimension $\mathscr{D}$ is process independent. 
The evolution equations allow one to resum rapidity logarithms and rewrite the Wilson coefficient in a more compact form

$$
\tilde{C}_{i \rightarrow j}=\exp \left[-\mathscr{D}^{i} \mathbf{L}_{\sqrt{\zeta}}\right] \tilde{\mathscr{C}}_{i \rightarrow j}
$$

The most general structure of $\tilde{\mathscr{C}}_{i j}$ of the $n$-th perturbative order is

$$
\tilde{\mathscr{C}}_{i j}^{[n]}=\sum_{k=0}^{2 n} \tilde{\mathscr{C}}_{i j}^{(n ; k)} \mathbf{L}_{\mu}^{k}
$$

The coefficients $\mathscr{C}^{(n ; k)}$ are related by the recursive relation

$$
(k+1) \tilde{\mathscr{C}}_{i \rightarrow j}^{(n ; k+1)}=\sum_{r=1}^{n} \frac{\Gamma_{\text {cusp }}^{[r]}}{2} \tilde{\mathscr{C}}_{i \rightarrow j}^{(n-r ; k-1)}-\frac{\gamma_{V}^{i[r]}-2(n-r) \beta^{[r]}}{2} \tilde{\mathscr{C}}_{i \rightarrow j}^{(n-r ; k)}-\tilde{\mathscr{C}}_{i \rightarrow k}^{(n-r ; k)} \otimes \frac{\mathscr{P}_{k \rightarrow j}^{[r]}}{z^{2}} \text {.(5.5) }
$$

Thus, given the expressions for the anomalous dimensions one needs only the boundary coefficients $\tilde{\mathscr{C}}^{(n, 0)}$ in order to reproduce the complete expression for the matching coefficient.

\section{Results}

To complete the discussion we present the explicit expressions for the quark TMD FF boundary terms up to NNLO. They are

$$
\begin{aligned}
& \tilde{\mathscr{C}}_{q \rightarrow q}^{(0 ; 0)}=\delta(\bar{z}), \\
& \tilde{\mathscr{C}}_{q \rightarrow q}^{(1 ; 0)}=\frac{C_{F}}{z^{2}}\left[(4 p(z) \ln z+2 \bar{z})_{+}+\delta(\bar{z})\left(6-\frac{3}{2} \pi^{2}\right)\right],
\end{aligned}
$$

where $p(z)=\frac{1+z^{2}}{1-z}$. The corresponding quark-antiquark coefficients are zero.

The NNLO coefficient is convenient to present in the following form

$$
\tilde{\mathscr{C}}_{q \rightarrow q}^{(2 ; 0)}(z)=C_{F}^{2} Q_{F}(z)+C_{F} C_{A} Q_{A}(z)+T_{R} N_{f} Q_{N}(z), \quad \tilde{\mathscr{C}}_{q \rightarrow \bar{q}}^{(2 ; 0)}(z)=C_{F}\left(C_{F}-\frac{C_{A}}{2}\right) Q_{q \bar{q}}(z)
$$

Then the functions $Q_{i}$ are

$$
\begin{aligned}
Q_{F}(z)= & \frac{1}{z^{2}}\left\{p ( z ) \left[104 \operatorname{Li}_{3}(z)-4 \operatorname{Li}_{3}(\bar{z})+4 \ln \bar{z} \operatorname{Li}_{2}(\bar{z})+48 \ln z \operatorname{Li}_{2}(\bar{z})-2 \ln ^{2} \bar{z} \ln z+82 \ln \bar{z} \ln ^{2} z\right.\right. \\
& \left.-24 \ln ^{3} z+\frac{39}{2} \ln ^{2} z-\left(8+\frac{44 \pi^{2}}{3}\right) \ln z-104 \zeta_{3}\right]+\bar{z}\left[-24 \operatorname{Li}_{2}(\bar{z})+4 \ln z \ln \bar{z}+42-\frac{\pi^{2}}{3}\right] \\
& \left.+9(1+z) \ln ^{3} z+\frac{25}{2}(-3+z) \ln ^{2} z+(-22+62 z) \ln z+2 \ln \bar{z}\right\}_{+} \\
& +\delta(\bar{z})\left(-\frac{13}{8}-\frac{55 \pi^{2}}{3}-12 \zeta_{3}+\frac{1037 \pi^{4}}{360}\right),
\end{aligned}
$$




$$
\begin{aligned}
& Q_{A}(z)= \frac{1}{z^{2}}\left\{p ( z ) \left[12 \mathrm{Li}_{3}(z)+4 \operatorname{Li}_{3}(\bar{z})-4 \ln \left(\frac{\bar{z}}{z^{2}}\right) \operatorname{Li}_{2}(\bar{z})+3 \ln ^{3} z+4 \ln \bar{z} \ln ^{2} z\right.\right. \\
&\left.-\frac{11}{6} \ln ^{2} z+\frac{10\left(7-\pi^{2}\right)}{3} \ln z+2 \zeta_{3}-\frac{404}{27}\right]+\bar{z}\left(4 \operatorname{Li}_{2}(\bar{z})-\frac{\pi^{2}}{3}+\frac{44}{3}\right) \\
&+\left.(8+2 z) \ln ^{2} z-2 \ln \bar{z}+\left(\frac{116}{3}-\frac{74}{3}\right) \ln z\right\}_{+}+\delta(\bar{z})\left(\frac{6353}{81}-\frac{443 \pi^{2}}{36}-\frac{278}{9} \zeta_{3}+\frac{91 \pi^{4}}{90}\right), \\
& Q_{N}(z)=\frac{1}{z^{2}}\left[\left(\frac{2}{3} \ln ^{2} z-\frac{20}{3} \ln z+\frac{112}{27}\right) p(z)-\frac{16}{3} \bar{z} \ln z-\frac{4}{3} \bar{z}\right]_{+}+\delta(\bar{z})\left(-\frac{2717}{162}+\frac{25 \pi^{2}}{9}+\frac{52}{9} \zeta_{3}\right), \\
& Q_{q \bar{q}}(z)= \frac{1}{z^{2}}\left\{p ( - z ) \left[8 \operatorname{Li}_{3}(-z)+16 \operatorname{Li}_{3}(z)-16 \operatorname{Li}_{3}\left(\frac{1}{1+z}\right)+8 \ln z\left(\operatorname{Li}_{2}(-z)-\operatorname{Li}_{2}(z)\right)\right.\right. \\
&\left.-6 \ln ^{3} z+\frac{8}{3} \ln ^{3}(1+z)+12 \ln ^{2} z \ln (1+z)-\frac{4 \pi^{2}}{3} \ln (1+z)+4 \zeta_{3}\right] \\
&-8\left(2+\operatorname{Li}_{2}(\bar{z})+8(1+z)\left(\operatorname{Li}_{2}(-z)+\ln z \ln (1+z)+\frac{\pi^{2}}{12}\right)\right. \\
&
\end{aligned}
$$

These expressions represent the main result of our calculation. The further details can be found in [20]

\section{Conclusion}

Using explicit definition of TMDFF as the product the collinear matrix element and the square root of the soft function in the coordinate space, we perform NNLO evaluation and analysis of TMDFF. The calculation performed within the standard QCD and in Feynman gauge, includes the independent computation of the soft function and the collinear matrix element, and their subsequent recombination into a TMD. This is a first calculation of individual TMDs at NNLO.

In order to perform the calculation we have reformulated the rapidity regularization of Ref. [U]]. The used regularization scheme (modified $\delta$-regularization scheme) is suitable for the multi-loop evaluation. Within this regularization we obtain the complete analytical expression for the TMDFF, and comprehensively investigate the structure of soft/rapidity singularities and their cancellation. The cancellation of singularities provides a strong check of the final result.

As a further check we find a complete agreement between the logarithmical part of the final result and the known predictions of renormalization group. The soft factor that has been evaluated in this work, is universal and spin-independent, and thus can be used for the calculation of all TMDs at NNLO. The explicit analytical expression for the soft factor allows us directly extract the function $\mathscr{D}\left(b_{T}\right)$ (the anomalous dimension of rapidity parameter evolution), and show it universality. We found complete agreement with known expressions for $\mathscr{D}\left(b_{T}\right)$ extracted from the analysis of Drell-Yan process. 
Finally, the calculation of the TMDFF performed in this work allows us to extract the relevant perturbative matching coefficient at NNLO, necessary to perform the resummation of large logarithms at NNNLL. The applied method can be readily used to obtain other relevant perturbative ingredients. The talk is bases on the ref.[20]].

Acknowledgements I would like to thanks organizers of QCD Evolution 2015 for invitation and support.

\section{References}

[1] J. C. Collins and D. E. Soper, Nucl. Phys. B 194 (1982) 445.

[2] J. C. Collins and D. E. Soper, Nucl. Phys. B 193 (1981) 381 [Nucl. Phys. B 213 (1983) 545].

[3] R. Angeles-Martinez et al., arXiv:1507.05267 [hep-ph].

[4] S. Catani and M. Grazzini, Eur. Phys. J. C 72 (2012) 2013 [Eur. Phys. J. C 72 (2012) 2132] [arXiv:1106.4652 [hep-ph]].

[5] S. Catani, L. Cieri, D. de Florian, G. Ferrera and M. Grazzini, Eur. Phys. J. C 72 (2012) 2195 [arXiv:1209.0158 [hep-ph]].

[6] S. Catani, L. Cieri, D. de Florian, G. Ferrera and M. Grazzini, Nucl. Phys. B8812014 414 [arXiv:1311.1654[hep-ph]].

[7] T. Gehrmann, T. Lubbert and L. L. Yang, Phys. Rev. Lett. 109 (2012) 242003 [arXiv:1209.0682 [hep-ph]].

[8] T. Gehrmann, T. Luebbert and L. L. Yang, JHEP 1406 (2014) 155 [arXiv:1403.6451 [hep-ph]].

[9] J. C. Collins, Foundations of perturbative QCD (Cambridge University Press, Cambridge, 2011).

[10] M. G. Echevarria, A. Idilbi and I. Scimemi, Phys. Lett. B 726 (2013) 795 [arXiv:1211.1947 [hep-ph]].

[11] M. G. Echevarria, A. Idilbi and I. Scimemi, Phys. Rev. D 90 (2014) 1, 014003 [arXiv:1402.0869 [hep-ph]].

[12] S. M. Aybat and T. C. Rogers, Phys. Rev. D 83 (2011) 114042 [arXiv:1101.5057 [hep-ph]].

[13] M. G. Echevarria, A. Idilbi and I. Scimemi, JHEP 1207 (2012) 002 [arXiv:1111.4996 [hep-ph]].

[14] A. A. Vladimirov, JHEP 1408 (2014) 089 [arXiv:1402.3182 [hep-ph]].

[15] A. Bacchetta and A. Prokudin, Nucl. Phys. B 875 (2013) 536 [arXiv:1303.2129 [hep-ph]].

[16] M. G. Echevarria, T. Kasemets, P. J. Mulders and C. Pisano, JHEP 1507 (2015) 158 [arXiv:1502.05354 [hep-ph]].

[17] R. Zhu, P. Sun and F. Yuan, Phys. Lett. B 727 (2013) 474 [arXiv:1309.0780 [hep-ph]].

[18] A. A. Vladimirov, JHEP 1506 (2015) 120 [arXiv:1501.03316 [hep-th]].

[19] I. O. Cherednikov and N. G. Stefanis, Nucl. Phys. B 802 (2008) 146 [arXiv:0802.2821 [hep-ph]].

[20] M. G. Echevarria, I. Scimemi, A.A.Vladimirov, arXiv:1509.06392 [hep-ph]. 